\title{
Radiochromic and radiofluorogenic solid state polymer dosimeter; a third signal: Electron Paramagnetic Resonance (EPR)
}

Bernal-Zamorano, M.R.; Sanders, N.H.; Lindvold, L.; Andersen, C.E.

Published in:

Radiation Physics and Chemistry

Link to article, DOI:

10.1016/j.radphyschem.2019.04.008

Publication date:

2019

Document Version

Peer reviewed version

Link back to DTU Orbit

Citation (APA):

Bernal-Zamorano, M. R., Sanders, N. H., Lindvold, L., \& Andersen, C. E. (2019). Radiochromic and radiofluorogenic solid state polymer dosimeter; a third signal: Electron Paramagnetic Resonance (EPR). Radiation Physics and Chemistry, 161, 72-76. https://doi.org/10.1016/j.radphyschem.2019.04.008

\section{General rights}

Copyright and moral rights for the publications made accessible in the public portal are retained by the authors and/or other copyright owners and it is a condition of accessing publications that users recognise and abide by the legal requirements associated with these rights.

- Users may download and print one copy of any publication from the public portal for the purpose of private study or research.

- You may not further distribute the material or use it for any profit-making activity or commercial gain

- You may freely distribute the URL identifying the publication in the public portal 


\section{Accepted Manuscript}

Radiochromic and radiofluorogenic solid state polymer dosimeter; a third signal:

Electron Paramagnetic Resonance (EPR)

M.R. Bernal-Zamorano, N.H. Sanders, L. Lindvold, C.E. Andersen

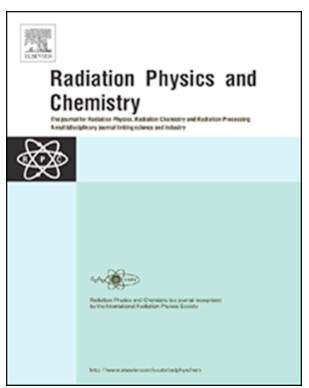

PII:

S0969-806X(18)31129-0

DOI:

https://doi.org/10.1016/j.radphyschem.2019.04.008

Reference: $\quad$ RPC 8258

To appear in: Radiation Physics and Chemistry

Received Date: 31 October 2018

Revised Date: 13 March 2019

Accepted Date: 6 April 2019

Please cite this article as: Bernal-Zamorano, M.R., Sanders, N.H., Lindvold, L., Andersen, C.E., Radiochromic and radiofluorogenic solid state polymer dosimeter; a third signal: Electron Paramagnetic Resonance (EPR), Radiation Physics and Chemistry (2019), doi: https://doi.org/10.1016/ j.radphyschem.2019.04.008.

This is a PDF file of an unedited manuscript that has been accepted for publication. As a service to our customers we are providing this early version of the manuscript. The manuscript will undergo copyediting, typesetting, and review of the resulting proof before it is published in its final form. Please note that during the production process errors may be discovered which could affect the content, and all legal disclaimers that apply to the journal pertain. 
Authors: M.R. Bernal-Zamorano ${ }^{\mathrm{a}}$, N.H. Sanders ${ }^{\mathrm{a}}$, L. Lindvold ${ }^{\mathrm{a}}$, C.E. Andersen ${ }^{\mathrm{a}}$.

${ }^{a}$ Center for Nuclear Technologies, Technical University of Denmark, Ris $\varnothing$ Campus, Frederiksborgvej 399, 4000 Roskilde, Denmark.

* Corresponding author.

E-mail: lali@dtu.dk

\title{
HIGHLIGHTS
}

- EPR signal from the dosimeter is detected and it increases with the dose, linearly for the medical dose range.

- Three methods for the solid polymer dosimeter's characterization: optical absorbance, fluorescence and EPR.

- $\quad$ EPR as supporting feature to the optical signals from the dosimeter.

- The measured EPR signal originates from radiation-induced changes of the radiochromic dye and not the matrix polymer.

\section{KEYWORDS}

3D dosimetry; solid-state polymer dosimeter; EPR; fluorescence; pararosaniline leuco dye, free radical.

\begin{abstract}
A solid-state polymeric dosimeter has been developed. The solid-state dye-doped polymeric dosimeter comprises the photo-curable polymer materials Poly(ethylene glycol)-diacrylate (PEGDA) and 2-Hydroxyethyl-methacrylate (HEMA) and the radiochromic leuco-dye 4,4',4'"- Triamino-triphenyl -acetonitrile. Using a photo-curable polymer blend facilitates a tight control of the polymerization process and hence the properties of the solid-state dosimeter. The radiation sensitive leuco-dye responds to ionising radiation by forming a stable free radical - a triphenylmethyl dye. This free radical has strong colour, and is fluorescent because it is dissolved in the solid polymeric material. Both physical properties can be measured optically. The free radical, however, provides a third signal as it is paramagnetic and hence can be detected by means of Electron Paramagnetic Resonance (EPR) spectroscopy. The EPR signal makes it possible to measure the radiation-induced free radical signal free from optical artefacts of the polymer material. Results from irradiations using a low-dose-rate cobalt-60 gamma irradiator shows an EPR signal measured using a Bruker EMX EPR spectrometer for the solid polymeric dosimeter, whose peak-to-peak amplitude is linear with the dose in the medical dose range and saturates for higher doses (100 Gy).
\end{abstract}

\section{Introduction}

The aim of this work is to develop a new method for making a solid-state polymeric fluorogenic dosimeter based on photo curing of a monomer matrix containing a radiation sensitive leuco-dye. Optical methods like spectrophotometry and laser-induced fluorescence will be used to detect the radiation-induced formation of the triphenylmethane free radical from the leuco-dye. These optical signals can be used to quantify the absorbed dose. The motivation for using the fluorescent properties of the triphenylmethyl dye is that is possibly could be used in solid-state dosimeters for dose-depth measurement of low energy electron beams and ultimately 3D medical dosimetry (Watanabe et al., 2017).

During the course of this development work, it became clear that a method for ascertaining a relationship between the absorbed dose and the formation of free radical dye was needed. A method for doing so is that of Electron Paramagnetic Resonance (EPR). EPR has previously been reported for quantifying free radical formation in dye solutions caused by UV irradiation (Brezová et al., 2004). The advantage of using the EPR signal is that it allows us to quantify how the matrix (polymer) affects the optical signals, absorption and fluorescence, from the dye. As we also employ a photoinitiator (triplet sensitizer) to facilitate the photo curing of the monomer blend containing the leuco-dye, the EPR signal allows us to monitor any influence on the dosimetric properties of the solidified (cured) polymer. 
Electron paramagnetic resonance (EPR) has also been used to study free radicals formed in solid materials like alanine. This technique is used to determine the absorbed dose in the amino acid alanine, which is commonly used as a reference dosimeter due to its water equivalence, high stability, low fading, and response over a high dose range (HeltHansen et al., 2009)(ICRU 80,2008). Established methods for alanine dosimetry, though, only give point doses (i.e. the averaged dose over the size of the detector pellet).

We presented in a previous work (Bernal-Zamorano et al., 2017a) a candidate material for 3D dosimetry, since the dosimeter is solid, water equivalent, and can be moulded into any shape based on the photocuring properties of the monomer blend used in this work. It is based on 4,4',4', - Triamino-triphenyl -acetonitrile leuco-dye, which is chemically transformed into a triphenyl methyl free radical by ionizing radiation (Fig. 1) (ICRU 80, 2008). This solidstate polymer dosimeter responds to ionizing radiation primarily by changes in its optical absorbance (as current 3D dosimeters do). The objective of this study was to make point detectors with this dosimeter to test if it is possible to measure the EPR signal as it is done with alanine. This would allow us to compare our dosimeter with a reference dosimeter, as it is usually done in dosimetry (Waldeland et al., 2011), and to be able to investigate the chemical changes and the mechanisms of this new dosimeter. It is also possible that the dosimeter will be useable via measurements of laser-stimulated fluorescence, which would be a more sensitive and faster method to obtain the absorbed dose point by point. However, this method requires that the background fluorescence of the polymeric material comprising the matrix of the solid-state dosimeter can be kept at a very low level.

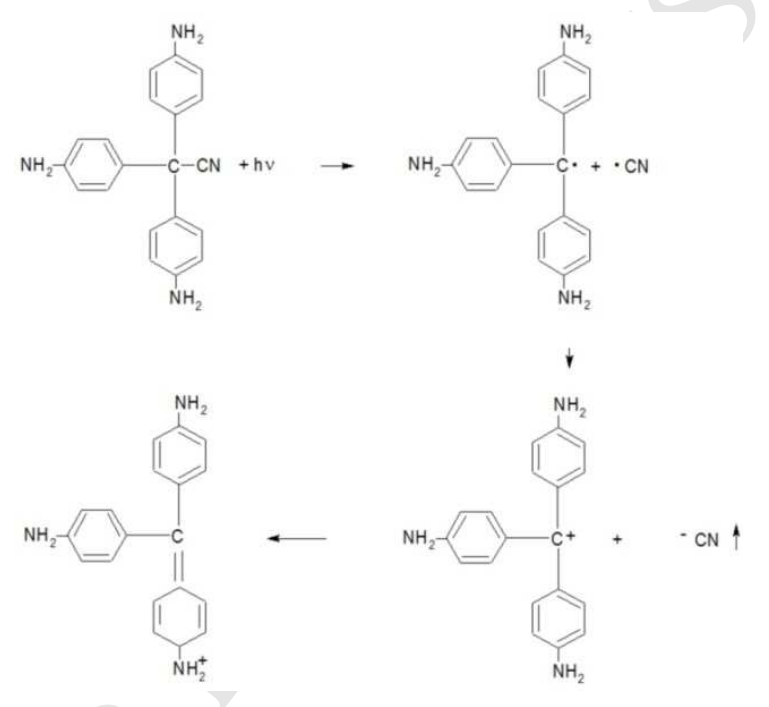

Fig.1. Chemistry involved in the response of the dye to radiation. Please note, that the cyano ion liberated by the radiochemical process is scavenged by the presence of a weak acid, typically citric or acetic acid in polymeric matrix.

\section{Materials and methods}

\subsection{Fabrication}

A Pararosaniline leuco dye, 4,4',4', - Triamino-triphenyl -acetonitrile (Clausson Kaas CK1467), is dissolved in a poly(ethylene glycol) diacrylate (PEGDA average $M_{n}$ 575) (Sigma Aldrich 437441) matrix that includes ethanol, 2hydroxyethyl methacrylate (HEMA) (Sigma Aldrich 128635), and the photoinitiator diphenyl(2,4,6-trimethylbenzoyl) phosphine oxide (TPO) (Sigma Aldrich 415952) for photocuring. The identical composition and procedure were used in a previous study (Bernal-Zamorano et al., 2017b) (Fig. 2a): Leuco-dye-61 mM, ethanol-0.83 M, PEGDA-1.62 M, HEMA-41 mM and TPO-1.41 mM. Solutions were mixed in a vortex rotor (Heidolph MultiReax) for half an hour before adding the photoinitiator and for half an hour more afterwards. They were kept in the darkness until the next day when they were photo-cured to form a solid state dosimeter and subsequently irradiated in our gammacell. Polymeric samples without the radiation-sensitive leuco-dye were made. The purpose of this experiment was to measure the baseline in optical absorption, fluorescence and EPR signal without the presence of the leuco-dye prior to and after gamma irradiation.

We made cylindrical pellets of $4.75 \mathrm{~mm}$ diameter and $2.78 \mathrm{~mm}$ thickness of the same size as the alanine pellets used in this work. We solidified the solution by using an aluminium mould between two glass plates to ensure the best 
a)
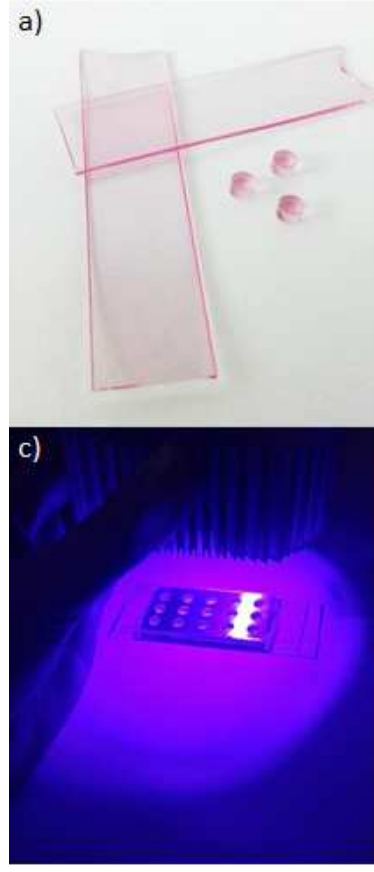

b)

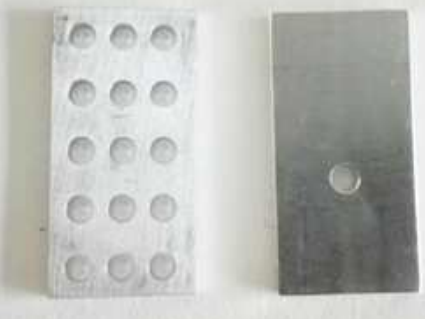

d)

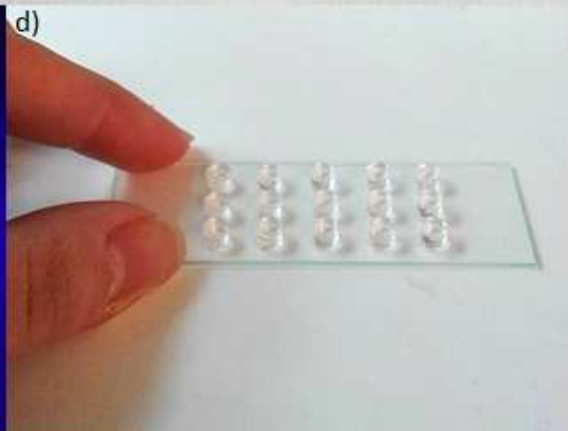

Fig.2. a. Slides and pellets of the dosimeter material. b. Aluminium mould for fabrication and holder for optical spectroscopy measurements. c. Photo-curing process with $407 \mathrm{~nm}$ LED. d. Result after curing.

\subsection{Irradiations and measurements}

Four pellets of each type (alanine, dosimeter and dosimeter's matrix) were irradiated in a ${ }^{60} \mathrm{Co}$ gamma cell at a dose rate of approximately $5 \mathrm{~Gy} \mathrm{~min}^{-1}$. The gamma cell contains a circular array of 8-rod sources that ensure homogeneous irradiation of samples placed in the middle of the source array. The pellets were given doses of: 5, 10, 20, 30, 50, 75 and 100 Gy. After each irradiation, the optical absorbance, fluorescence and EPR signals of each pellet of the dosimeter and its matrix without leuco-dye were measured. The EPR signal of alanine pellets was also measured. Fig. 3 shows the pellets and the holders that were used.

Optical absorbance spectra of the pellets were measured with a Shimazdu UV-2700 spectrophotometer. The fluorescence spectra from the pellets were obtained by excitation of the pellets with a collimated $520 \mathrm{~nm}$ diode laser and an Ocean Optics QE6500 CCD spectrograph to record the spectra (Højer Sanders, 2017). The EPR signal was obtained using a Bruker EMX spectrometer by inserting each pellet into the resonator in a quartz tube. Before taking each measurement, fine tuning of the cavity was performed. Peak-to-peak amplitude of the first derivative EPR spectrum for alanine is obtained directly through the Risø EPR user interface. In the case of the new dosimeter, peaks are obtained by fitting the maximum of the peak-to-peak amplitude of each first derivative EPR spectrum to a fourth-order polynomial, separately. The magnetic sweep width was $50 \mathrm{G}$ with a resolution of 1024 points, the modulation amplitude was $1 \mathrm{G}$, the frequency $100 \mathrm{kHz}$, the sweep time $5.24 \mathrm{~s}$ and the time constant $1.25 \mathrm{~ms}$. The microwave frequency was $9.75 \mathrm{GHz}$. Measuring the EPR signal from the pararosaniline dye, constituting the free radical causing the intense magenta colour and red fluorescence, is challenging. As the dye is dissolved in a solid polymeric matrix made of two hydrophilic polymers, also used in making soft contact lenses, a few percent of water is present in the matrix. The presence of water is required for the radiation-induced transformation of leuco-dye into the coloured dye to take place. The water, however, poses a challenge when measuring the EPR signal from the polymeric pellet as the water attenuates the 9.75 $\mathrm{GHz}$ microwave probe signal in the cavity of the EPR. This challenge, however, can be resolved by careful tuning of the cavity. 


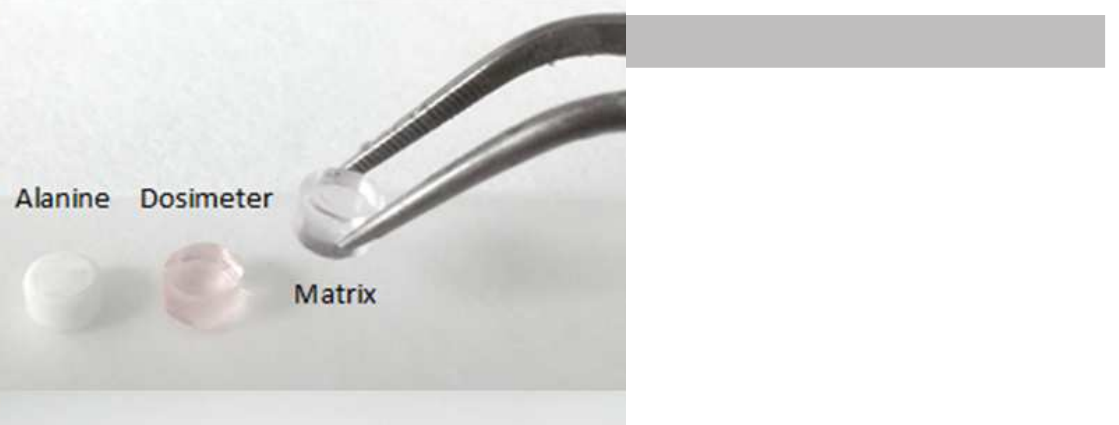

b)

Fig.3. a. Pellets of alanine, solid polymer dosimeter and matrix of the dosimeter (no dye). b. Mould for fabrication, holder for optical spectroscopy and poly(ethyl ether ketone) (PEEK) holder for irradiation of 4 pellets.

\section{Results and discussion}

Fig. 4 shows the results of the EPR of alanine pellets and Fig. 5 shows the results of the EPR, absorbance and fluorescence signals of the new dosimeter and its matrix. Table 1 collects the results with the corresponding uncertainties.
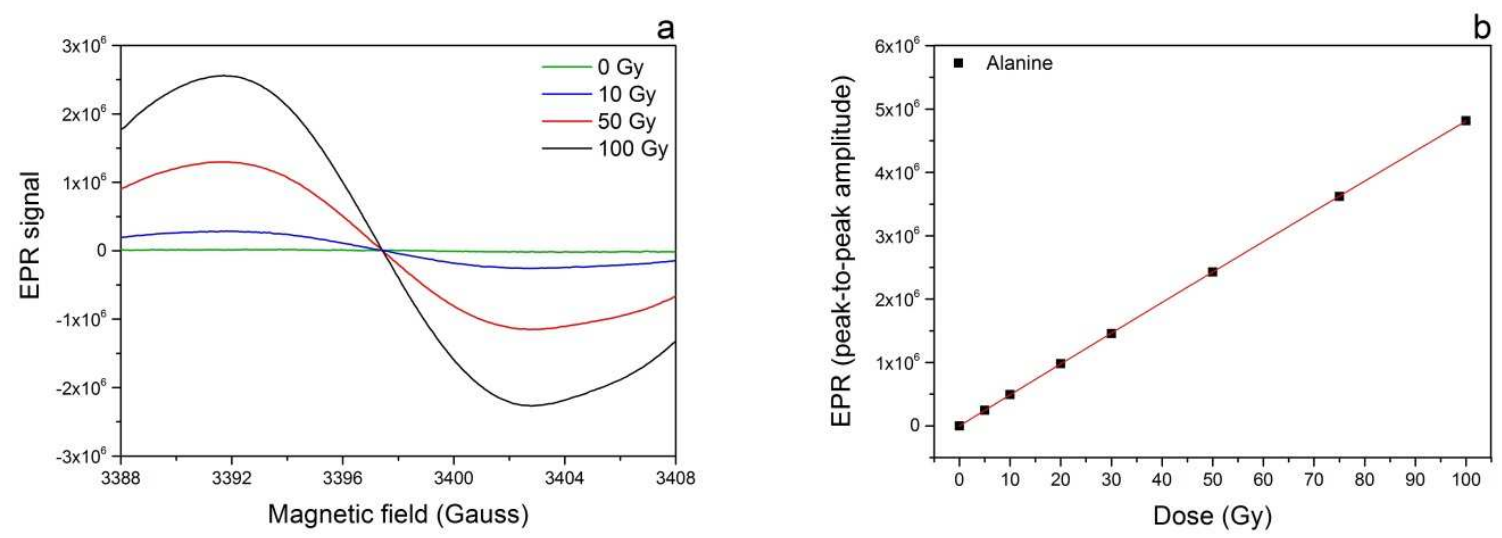

Fig. 4: (a) EPR signal (first derivative of the EPR absorption spectra) of an alanine pellet irradiated to several doses, and (b) peak-to-peak amplitude of the EPR signal as function of the dose for 4 alanine pellets (b). Error bars representing the statistical spread out of the measurements of the 4 pellets (standard deviation) are smaller than the markers.

\subsection{EPR signal}

A clear EPR signal was obtained for our material (Fig. 5a), increasing with the dose. As presented in (BernalZamorano et al., 2017b), alanine and PEGDA have similar characteristics in terms of its water equivalence (similar effective atomic number, mass density and electronic density). Simulations using Monte-Carlo (EGS_CHAMBER) has been made (Bernal-Zamorano, 2018), to calculate dose ratio $\mathrm{D}_{\text {dosimeter }} / \mathrm{D}_{\text {water }}$ for different mono-energetic photon beams in the energy range $0.1-20 \mathrm{MeV}$. In the clinical relevant range $1-20 \mathrm{MeV}$, a ratio of 0.96 was calculated for the dyedoped polymeric solid-state dosimeter presented in this paper showing an energy independent response in this region. This indicates that the material can be regarded as water equivalent for energy range of mono-energetic photons.

Fig. $5 \mathrm{~b}$ shows that the new dosimeter material tends to saturate above $20 \mathrm{~Gy}$, which is not the case for alanine (Fig. 4). This difference is probably due to the interaction of the un-paired electron from the free radical with its environment, which affects the details of the EPR spectrum. While alanine pellets are alanine microcrystals contained in 
compact wax, the new dosimeter material is similar to tissue: compact on the surface but diffusive inside. As indicated by its chemical composition formula, the new dosimeter material contains free water molecules that may move within the dosimeter's matrix by diffusion processes, and bound water, which is chemically attached to other molecules. Therefore, since water has a strong absorption band in the microwave region (Mehdizadeh, 2009) at the microwave frequency used here $(9.75 \mathrm{GHz})$, most energy is absorbed by the dosimeter, resulting in a saturation of the EPR signal from the dye. However, despite this earlier saturation compared to alanine, the EPR signal of the new dosimeter material is approximately linear for medical doses, which is likely to be the main application for this dosimeter.

The saturation dose can be obtained by fitting the peak-to-peak amplitude of the EPR spectra to Eq. (1) (Waldeland et al., 2011) (Rotblat and Simmons, 1963), where $N(D)$ is the number of free radicals as function of the dose, $N_{\infty}$ is the number of radicals at saturation, and $D_{0}$ is the characteristic saturation dose of the dosimeter. By fitting the dosimeter curve in Fig.5b (but with background correction; data starting in the origin) the saturation dose was obtained, resulting in 48.1 Gy with standard deviation $6 \%$.

$$
N(D)=N_{\infty}\left(1-e^{-\frac{D}{D_{0}}}\right) \quad \text { Eq. (1) }
$$

Following Eq. (1), the peak-to-peak amplitude of the first derivative of the EPR signal provides a measurement of the free radicals present in the dosimeter. We have identified that these free radicals are only present in the dosimeter with dye and not in the matrix without it (Fig. 5b). The curve for the matrix is flat following the first irradiation, since from 0 to 5 Gy the EPR peak-to-peak amplitude decreases by 53\%. This decrease is due to the remaining radical species from the photoinitiator, which have not completely disappeared after curing and they disappear with the first irradiation. Therefore, the dose response of the dosimeter is only due to the dye. It is important to ascertain that the matrix is not liberating free radicals that respond to radiation and that may interact with those from the dye.

\subsection{Optical absorbance and fluorescence signals}

From the optical absorbance results, in Fig.5d we see how the absorbance spectra are well differentiated for as the absorbed dose increases. This leads to a linear response when the absorption peaks are plotted against the absorbed dose for the entire dose range (Fig.5e). For the fluorescence, the spectra are less spread out, which decreases sensitivity. The background fluorescence, however, is very high, which makes it indistinguishable from the responses for low doses (Fig.5d, f). In Fig. $5 \mathrm{f}$ we also observe that the fluorescence of the dosimeter decreases with the first irradiation. As the photoinitiator itself is fluorescent, this decrease may be related to radiation-induced decomposition of the photoinitiator species with the first irradiation, observed in the EPR signal from the matrix. Therefore, the role of the matrix in the fluorescence response of the dosimeter is very important and the EPR signal helps us in studying it in more detail.
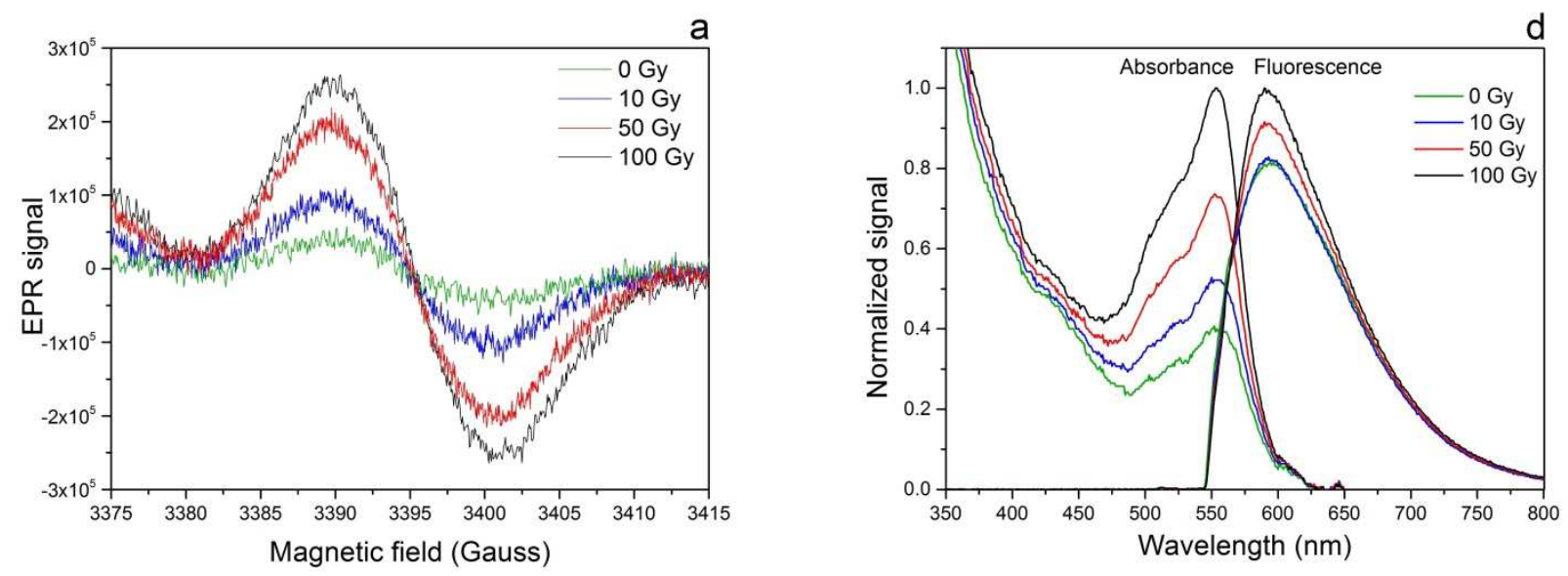

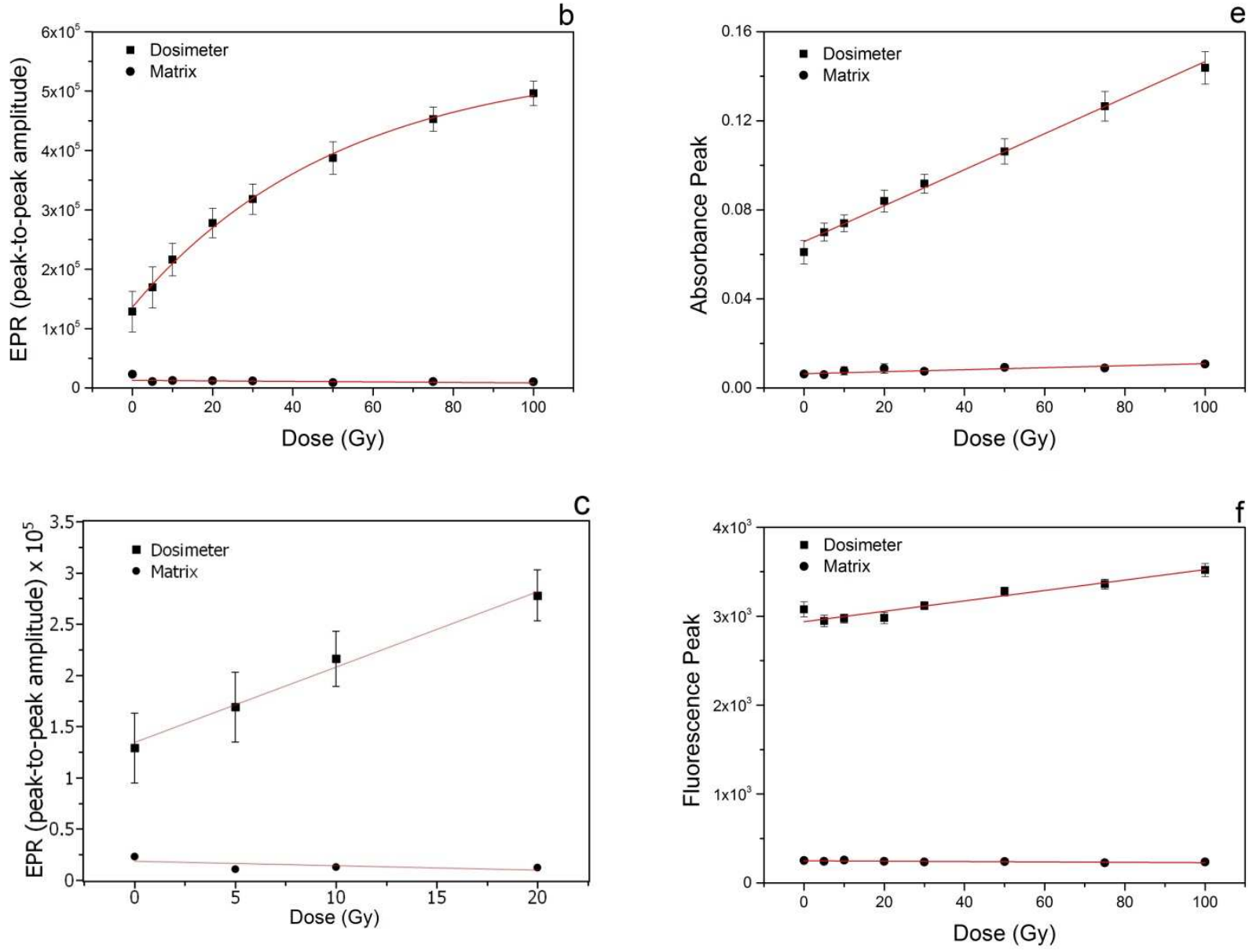

Fig. 5. (a) EPR signal (first derivative of the EPR absorption spectra) of a pellet of the dosimeter irradiated at several doses. (b) Peak-to-peak amplitude of the EPR signal of the dosimeter (squares) and of the dosimeter's matrix (dots) as function of the dose, with fittings. (c) Detail of figure $5 \mathrm{~b}$ in the 0-20 Gy range. (d). Optical absorbance and fluorescence spectra of a pellet of the dosimeter for several doses. (e) and (f) Absorbance and fluorescence peaks as function of the dose for the dosimeter (squares) and the dosimeter's matrix (dots), with fittings. Error bars represent the standard deviation of the measurements of the four pellets, and in some cases are smaller than the markers

\section{Table 1}

Values of Fig.5b, e and $\mathrm{f}$ with their correspondent standard deviation $(\sigma)$.

\begin{tabular}{ccccccc}
\hline \multirow{2}{*}{ Dose $(\mathbf{G y})$} & \multicolumn{2}{c}{ EPR $(\mathbf{x ~ 1 0})$} & \multicolumn{2}{c}{ Absorbance peak $\left(\mathbf{x ~ 1 0}^{-3}\right)$} & \multicolumn{2}{c}{ Fluorescence peak $\left.(\mathbf{x ~ 1 0})^{3}\right)$} \\
& Dosimeter & Matrix & Dosimeter & Matrix & Dosimeter & Matrix \\
\cline { 2 - 7 } $\mathbf{0}$ & $1.29(0.34)$ & $0.232(0.022)$ & $61.0(5.2)$ & $6.25(0.83)$ & $3.077(0.085)$ & $0.252(0.013)$ \\
$\mathbf{5}$ & $1.69(0.34)$ & $0.109(0.012)$ & $70.0(4.1)$ & $6.00(0.71)$ & $2.946(0.064)$ & $0.244(0.010)$ \\
$\mathbf{1 0}$ & $2.16(0.27)$ & $0.126(0.021)$ & $74.0(3.7)$ & $7.8(1.8)$ & $2.974(0.049)$ & $0.2560(0.0076)$ \\
$\mathbf{2 0}$ & $2.78(0.25)$ & $0.121(0.012)$ & $84.0(4.9)$ & $8.8(2.2)$ & $2.980(0.063)$ & $0.2426(0.0069)$ \\
$\mathbf{3 0}$ & $3.18(0.26)$ & $0.119(0.019)$ & $91.8(4.3)$ & $7.5(1.1)$ & $3.118(0.045)$ & $0.2359(0.0089)$ \\
$\mathbf{5 0}$ & $3.87(0.27)$ & $0.0895(0.0082)$ & $106.3(5.6)$ & $9.25(0.43)$ & $3.278(0.050)$ & $0.2403(0.0093)$ \\
$\mathbf{7 5}$ & $4.53(0.20)$ & $0.1064(0.0093)$ & $126.5(6.7)$ & $9.00(0.71)$ & $3.360(0.057)$ & $0.2258(0.0098)$ \\
$\mathbf{1 0 0}$ & $4.96(0.21)$ & $0.105(0.018)$ & $143.8(7.3)$ & $10.75(0.83)$ & $3.518(0.073)$ & $0.236(0.010)$ \\
\hline
\end{tabular}

\section{Conclusions}

Three signals have been measured for the dosimeter's characterization: optical absorbance, EPR, and laserstimulated fluorescence. 
The EPR signal from the solid-state polymer dosimeter has been measured, and increases linearly with the dose in the medical dose range below about $5 \mathrm{~Gy}$, approaching saturation for higher doses around $100 \mathrm{~Gy}$. The free radicals concentration measured via the EPR in the dosimeter do not arise from the irradiated matrix, but from the radiochromic dye. Therefore, the matrix provides the required properties for the dosimeter, such as solid support, water equivalence, mouldable, flexibility, optical clarity, good mechanical and optical properties, without interfering in the radiation response from the dye.

EPR signal is a source of improved understanding of the underlying dosimetric characteristics of this material and a supporting feature to the optical signals from the dosimeter.

Besides, interesting applications in particle therapy beams are anticipated as the signal production in solid-state dosimeters are generally dependent on the ionization density.

\section{Acknowledgements}

Mark Bailey, Torben E. Mølholt and Arne Miller for their help with the EPR technique. Søren V. Dalsgaard and Jan S. Andersen for their help with the fabrication of aluminium mould.

\section{References}

Bernal-Zamorano M.R., Sanders N.H., Lindvold L., Andersen C.E. (2017a). Radiochromic and radiofluorogenic 3D solid polymer dosimeter: initial results for high doses. Journal of Physics: Conference Series, Vol. 847, 012016, DOI: 10.1088/1742-6596/847/1/012016.

Bernal-Zamorano M.R., Sanders N.H., Lindvold L., Andersen C.E. (2017b). Radiochromic and radiofluorogenic 3D solid polymer dosimeter; effect of the photoinitiator. Radiation Measurements, DOI:10.1016/j.radmeas.2017.03.012.

Bernal-Zamorano M.R, (2018), Development and characterization of radiochromic and radiofluorogenic solid state polymer dosimeter material, PhD-thesis, Technical University of Denmark

Brezová V., Pigošová J., Havlinová B., Dvoranová D., Ďurovič M.. (2004), EPR study of photochemical transformations of triarylmethane dyes, Dyes and Pigments, Vol. 61, 177-198, DOI:10.1016/j.dyepig.2003.10.012

Helt-Hansen J., Rosendal F., Kofoed I.M., Andersen C.E. (2009). Medical reference dosimetry using EPR measurements of alanine: Development of an improved method for clinical dose levels. Acta Oncologica, 48:2, 216-222, DOI: 10.1080/02841860802279725.

Højer Sanders N., (2017), Towards 3D Dosimetry with Optical FluorescenceTomography Using a Radiofluorogenic Material: Setup and Data Processing, PhD Thesis, Technical University of Denmark

ICRU (2008). Journal of the ICRU Vol 8 No 2 Report 80.

Mehdizadeh M. (2009). Microwave/RF Applicators and Probes for Material Heating, Sensing, and Plasma Generation. Chapter 1. Elsevier.

Rotblat, J., Simmons, J.A. (1963). Dose-response relationship in yield of radiationinduced free radicals in amino acids. Physics in Medicine and Biology 7, 489e497.

Waldeland E., Helt-Hansen J., Malinen E. (2011). Characterization of lithium formate EPR dosimeters for high dose applications - Comparison with alanine. Radiation Measurements, DOI: 10.1016/j.radmeas.2010.11.015.

Watanabe Y., Warmington L., Gopishankar N. (2017). Three-dimensional radiation dosimetry using polymer gel and solid radiochromic polymer: From basics to clinical applications. World Journal of Radiology, DOI: 10.4329/wjr.v9.i3.112. 